\title{
ADJOINT REGULAR RINGS
}

\author{
HENRY E. HEATHERLY and RALPH P. TUCCI
}

Received 10 September 2001

Let $R$ be a ring. The circle operation is the operation $a \circ b=a+b-a b$, for all $a, b \in R$. This operation gives rise to a semigroup called the adjoint semigroup or circle semigroup of $R$. We investigate rings in which the adjoint semigroup is regular. Examples are given which illustrate and delimit the theory developed.

2000 Mathematics Subject Classification: 16N20, 16U99, 20M17, 20M18, $20 \mathrm{M} 19$.

1. Introduction. This paper continues the authors' investigation of adjoint semigroups of rings $[13,14]$. Here $R$ will always denote a ring (not necessarily commutative and not necessarily with unity). The Jacobson circle or adjoint operation, $a \circ b=$ $a+b-a b$, for each $a, b \in R$, yields a monoid $(R, \circ)$, the adjoint semigroup of $R$. Here we primarily consider the situation where $R$ is a (von Neumann) regular ring or where $(R, \circ)$ is (von Neumann) regular. Previous work along these lines has been done by Du [7] and Clark [3]. Our viewpoint is that of the interplay between semigroup properties of $(R, \circ)$ or $(R, \cdot)$ and ring properties of $R$.

For $x \in R$, we use $\mathbf{l}_{R}(x)$ and $\mathbf{r}_{R}(x)$ for the left and right annihilator sets of $x$ in $R$, respectively. When no ambiguity will arise, we use simply $\mathbf{l}(x)$ and $\mathbf{r}(x)$. The Jacobson radical of $R$ is denoted by $\mathscr{g}(R)$.

Let $S$ be a semigroup. We use $E(S)$ for the set of idempotents in $S$ and $Z(S)$ for the center of $S$. Frequent use will be made of the fact that $E(R, \cdot)=E(R, \circ)$ and $Z(R, \cdot)=$ $Z(R, \circ)$. Consequently we use $E(R)$ and $Z(R)$ for these sets, respectively.

Of particular interest here are the following types of regular semigroups. Let $T$ be a regular semigroup. If the idempotents of $T$ commute among one another, $T$ is said to be inverse; this is equivalent to the condition that the von Neumann inverse of each element in $T$ is unique, [5, Theorem 4.11]. If the idempotents of $T$ are central, then $T$ is said to be Clifford. It is well known that a Clifford semigroup is a union of groups, [5, Theorem 1.17]. If the idempotents form a subsemigroup, then $T$ is called orthodox. If each element commutes with one of its von Neumann pseudoinverses, then $T$ is completely regular. This last condition is equivalent to the condition that $T$ is a union of groups, [19, Theorem II.1.4].

Let $\mathscr{P}$ be a semigroup property (or from another vantage point, $\mathscr{P}$ could be thought of as a class of semigroups closed under isomorphism). If $(R, \cdot)$ has property $\mathscr{P}$ we say $R$ is a $\mathscr{P}$-ring, and if $(R, \circ)$ has property $\mathscr{P}$ we say $R$ is an adjoint $\mathscr{P}$-ring. Exemplary of such properties are regular, completely regular, or Clifford. It is worth noting that what here is called an adjoint completely regular ring is called a generalized radical ring in [3, 8]. (For terminology and basic facts on semigroups, see [5] or [19].) 
2. Preliminaries. In this section, we present some preliminary results. Let $R^{1}$ be the standard Dorroh extension of a ring $R$ to the ring $R^{1}$, which has unity. Recall that this embeds $R$ as an ideal of $R^{1}$, so we can identify $R$ as the ideal $\bar{R}$ in $R^{1}$. We will at times use $R^{1}$ in conjunction with $R$ in order to make use of the simplifying attributes of having an identity for calculation. Recall that the mapping $\phi: x \rightarrow 1-x$ is an isomorphism from $\left(R^{1}, \circ\right)$ onto $\left(R^{1}, \cdot\right)$, and $\phi$ restricted to $R$ yields an injective homomorphism. Observe that if $R$ has identity, then this same mapping, $x \rightarrow 1-x$, yields an isomorphism from $(R, \circ)$ onto $(R, \cdot)$. This observation makes the next result immediate. (See also [9, Lemma 20].)

Proposition 2.1. Let $a, b \in R$. Then

(a) $a \circ b \in E(R)$ if and only if $(1-a)(1-b)=1-(a \circ b)$;

(b) $a \circ b \circ a=a$ if and only if $(1-a)(1-b)(1-a)=(1-a)$;

(c) $a \circ R=b \circ R$ if and only if $(1-a) R^{1}=(1-b) R^{1}$;

(d) $a$ is adjoint regular in $R$ if and only if $1-a$ is a regular element in $R^{1}$.

The next result is immediate, but it is useful enough to warrant stating.

Proposition 2.2. An element $a \in R$ is adjoint regular if and only if there exist $b \in R, e=e^{2} \in R$ such that $a+b-a b=e$ and $e a=e$.

Note that a quasi-regular element satisfies the conditions of Proposition 2.2 with $e=0$. Hence, the conditions for adjoint regularity are a natural generalization of the condition for quasi-regularity.

Du [7, Theorem 1] has shown that if $R$ is a regular ring, then $(R, \circ)$ is a regular monoid. We next give a different proof of that result.

PRoposition 2.3. If $R$ is a regular ring, then $(R, \circ)$ is a regular monoid.

Proof. There is an injective ring homomorphism, $\phi: R \rightarrow R^{*}$, that embeds $R$ as an ideal, $\hat{R}$, in the regular ring $R^{*}$ which has identity [10, Theorem 1]. Consequently, $\phi$ : $(R, \circ) \rightarrow\left(R^{*}, \circ\right)$ is an isomorphism. Since $R^{*}$ has identity we have that $\left(R^{*}, \cdot\right) \cong\left(R^{*}, \circ\right)$ and that each ideal of $\left(R^{*}, \cdot\right)$ is regular. So each ideal of $\left(R^{*}, \circ\right)$ is regular. Hence $(\hat{R}, \circ)$ is regular and so is its isomorphic image $(R, \circ)$.

Since every ideal of a regular semigroup is regular, we immediately have that if $R$ is a regular ring and $X$ is an ideal of $(R, \circ)$, then $(X, \circ)$ is regular.

By using the powerful Fuchs-Halperin result, this proof completely bypasses the calculations used in Du's proof [7]. Also, using similar methods, we can obtain analogous results by replacing the term regular in Proposition 2.3 by orthodox or inverse. Finally, note that the converse of Proposition 2.3 does not hold, as any example of a Jacobson radical ring will illustrate.

3. Equivalent conditions under regularity. In this section, we discuss various equivalent conditions to $R$ strongly regular and to $R$ adjoint completely regular. Examples are given which illustrate these results and show limitations to extending them.

It is well known that the following conditions are equivalent for a ring $R$ :

(1) for each $a \in R$ there exists $b \in R$ such that $a=a^{2} b$; 
(2) for each $a \in R$ there exists $c \in R$ such that $a=c^{2} a$;

(3) $R$ is regular and $E(R) \subseteq Z(R)$.

A ring $R$ satisfying any of (and hence all of) these conditions is called a strongly regular ring, [6]. Note that these conditions are not equivalent for semigroups, [19, Theorem II.1.4]. The next proposition ties strongly regular with several other conditions on the multiplicative semigroup of a ring. The proposition is a compilation of results from various sources. First some terminology is needed.

Following [17] we say that a semigroup $S$ is E-solid if, whenever $e, f, g \in E(S)$ such that $e \mathscr{L} f \mathscr{R} g$, there exists $h \in E(S)$ such that $e \mathscr{R} h \mathscr{L} \mathscr{g}$. (Here $\mathscr{L}$ and $\mathscr{R}$ are the standard Green's relations, [5, page 47].) Recall that the core of $S$, denoted by $C(S)$, is the subsemigroup of $S$ generated by $E(S)$, [19, page 89]. So $S$ is orthodox if and only if $S$ is regular and $E(S)=C(S)$. Let $\mathscr{P}$ be a semigroup property. Then $S$ is locally- $\mathscr{P}$ if $e S e$ has property $\mathscr{P}$ for every $e \in E(S)$.

Proposition 3.1. Let $R$ be a regular ring. The following are equivalent:
(a) $R$ is strongly regular;
(b) $R$ is orthodox;
(c) $R$ is completely regular;
(d) $R$ is inverse;
(e) $R$ is Clifford;
(f) $R$ is locally inverse;
(g) $R$ is E-solid;
(h) $R$ is locally E-solid;
(i) $C(R)$ is completely regular.

Proof. As mentioned above, (a) $\Leftrightarrow(\mathrm{e})$. So $(\mathrm{e}) \Rightarrow(\mathrm{b})$ is trivial, while (b) $\Rightarrow(\mathrm{e})$ follows from [22, Remark 15]. It is known that (a) $\Leftrightarrow(\mathrm{c})$, even for semigroups [19, page 58]. Next, (d) $\Leftrightarrow($ e) comes from [13], while $(\mathrm{d}) \Leftrightarrow(\mathrm{f}) \Leftrightarrow(\mathrm{g}) \Leftrightarrow(\mathrm{h})$ comes from [17], and (g) $\Leftrightarrow(\mathrm{i})$ comes from [12, Theorem 3]. This completes the logical circuit.

This by no means exhausts the vast number of known equivalent conditions to $R$ strongly regular, but it suffices for our purposes and gives a good sample of what is known. For more equivalent conditions to $R$ strongly regular see $[5,6,11,16,18,19$, $21,22]$.

We next consider various equivalent conditions on the adjoint semigroup of a regular ring.

PROPOSITION 3.2. Let $R$ be regular. The following are equivalent:

(a) $R$ is adjoint completely regular;

(b) $R$ is adjoint Clifford;

(c) $R$ is strongly regular.

Proof. Assume (a). Du [8, Lemma 11$]$ has shown that $(R, \circ)$ completely regular implies that $E(R)$ is closed under the ring multiplication. Since $R$ is regular, this yields that $R$ is orthodox. Thus $R$ is Clifford and by Proposition $3.1 R$ is strongly regular. Consequently, $R$ is adjoint regular. Conversely, $R$ regular and adjoint Clifford implies that $R$ is Clifford, and hence $R$ is strongly regular. We have established (a) $\Rightarrow(\mathrm{c}) \Leftrightarrow(\mathrm{b})$. Since 
a Clifford semigroup is a union of groups, $(R, \circ)$ Clifford implies $(R, \circ)$ completely regular, yielding $(b) \Rightarrow(a)$ and finishing the logical chain.

It is known [13] that $R$ is adjoint inverse if and only if $R$ is adjoint Clifford. Recently $\mathrm{Du}$ [9] has shown that adjoint orthodox implies adjoint completely regular. However, an adjoint completely regular ring need not be adjoint Clifford, as the next example shows.

EXAMPLE 3.3. Let $S$ be a right zero semigroup with more than one element and let $R$ be the semigroup ring $\mathbb{Z}_{2}[S]$. So every nonzero element of $R$ has the form $x=$ $e_{1}+\cdots+e_{n}$, for some distinct $e_{1}, \ldots, e_{n} \in S$. Let $y=f_{1}+\cdots+f_{m}$, where $f_{1}, \ldots, f_{m}$ are distinct terms from $S$. Then $x y=n y$. So $x y=0$, if $n$ is even, and $x y=y$, if $n$ is odd. Then $x$ is an idempotent if and only if $n$ is odd, and if $y$ is also an idempotent, then $x y=y$. Consequently $E(R)$ is closed under ring multiplication. Observe that $R=N(R) \cup E(R)$; hence $R$ is adjoint orthodox. Since elements in $E(R)$ are completely regular in $(R, \circ)$, and since elements in $N(R)$ are quasi-regular in $R$, and hence are completely regular in $R$, we have that $R$ is adjoint completely regular. However, since $\left(e_{1}+e_{2}\right) y\left(e_{1}+e_{2}\right)=0$, for each $y \in R$, we see that $R$ is not regular. Also, $R$ is not a Jacobson radical ring. Since $e_{1} e_{2} \neq e_{2} e_{1}$, for distinct $e_{1}, e_{2} \in S$, we see that $e_{1} \circ e_{2} \neq e_{2} \circ e_{1}$, and hence $R$ is not adjoint inverse.

Having $R$ regular implies that $R$ is adjoint regular, as we have seen. However, $R$ being regular does not imply that $R$ is adjoint inverse, adjoint completely regular, nor adjoint orthodox, as the next example illustrates.

EXAMPLE 3.4. Let $A$ be a regular ring with identity and let $R=M_{2}(A)$, the ring of $2 \times 2$ matrices over $A$. So $R$ is regular and hence $R$ is adjoint regular. But there are noncommuting idempotents in $R$; so $R$ is not adjoint inverse, and hence not adjoint completely regular by Proposition 3.2. By [9, Theorem 14], $R$ is not adjoint orthodox.

4. Decomposition. Let $R$ be a ring, and let $S_{1}, \ldots, S_{n}$ be subrings of $R$. If $R=S_{1}+$ $\cdots+S_{n}$ and whenever $s_{i} \in S_{i}, i=1, \ldots, n$ such that $s_{1}+\cdots+s_{n}=0$, then $s_{i}=0$, $i=1, \ldots, n$, then we say that $R$ is a supplementary sum of the $S_{i}, i=1, \ldots, n,[1]$. This is equivalent to $R^{+}=\sum_{i=1}^{n} \oplus S_{i}^{+}$, as a direct sum of abelian groups. We write $R=S_{1}+\cdots+S_{n}$ for such a supplementary sum. We next state as a lemma the wellknown two-sided Peirce decomposition, given here without using an identity in the ring. (See $[1,15]$.)

LEMMA 4.1. Let $e \in E(R)$. Then $R=e R e+e \cdot \mathbf{l}_{R}(e)+\mathbf{r}_{R}(e) \cdot e+\mathbf{r}_{R}(e) \cap \mathbf{l}_{R}(e)$.

Recall [2] that $e \in E(R)$ is said to be a left semicentral idempotent in $R$ if $e$ Re $=R e$, (equivalently, ere $=r e$, for each $r \in R$ ). It is well known that in this case $\mathbf{l}_{R}(e)$ is an ideal of $R$ and $R / \mathbf{l}_{R}(e) \cong e R e$.

Proposition 4.2. Let e be a left semicentral idempotent in $R$.

(a) $e R e=R e$ is a left ideal of $R ; l_{R}(e)=l_{R}(R e)$ is an ideal of $R$; and $e \cdot l_{R}(e)$ is a right ideal of $R$; 
(b) $R=R e \oplus_{l} l_{R}(R e)$ as a direct sum of left ideals of $R$ and $R / l_{R}(e) \cong e R e$, a ring with unity $e$;

(c) $e R=R e+e \cdot l_{R}(R e)$.

Proof. By definition, $R e=e R e$. The rest is routine.

In a strictly analogous fashion, we define right semicentral idempotent and obtain a dual result. For a central idempotent we naturally obtain a stronger result.

COROLlary 4.3. If e is a central idempotent in $R$, then $R=e R e \oplus \operatorname{Ann}_{R}(e R e)$, as a direct sum of ideals of $R$.

Following Clark and Lewin [4], $e \in E(R)$ is said to be a principal idempotent in $R$ if the homomorphic image of $e$ in $\bar{R}=R / J(R)$ is the identity in $\bar{R}$. This implies that if $u \in E(R)$ such that $e u=0=u e$, then $u=0$. (The latter condition, together with $e \neq 0$, is what Albert used in defining the principal idempotent [1, page 25].)

Principal idempotents play a key role in our next decomposition, whose proof makes use of the following result due to Du [7, Corollary 2].

LEMMA 4.4. If $R$ is adjoint regular and e is a principal idempotent in $R$, then $R=$ $e R e+J(R)$, as a supplementary sum of subrings.

We are now ready to give a much shorter proof of the main result in [3, Theorem B].

Proposition 4.5. Let e be an idempotent in a ring $R$. The following are equivalent:

(a) $R$ is adjoint completely regular and e is a principal idempotent in $R$;

(b) $R=e R e+J(R)$ and eRe is a strongly regular ring.

Proof. Assume (a). By Lemma 4.4, $R=e R e+J(R)$; so $R / J(R) \cong e R e$. Since the ring $e$ Re inherits the adjoint completely regular condition from $R$ and $e R e$ is regular, by Proposition 3.2 we have that ring eRe is strongly regular.

Assume (b). Then $e$ Re is adjoint regular, so by [7] the ring $R$ is adjoint regular. Since all the idempotents of $R$ are central, $R$ is adjoint completely regular. Clearly $e$ is a principal idempotent of $R$.

In view of Proposition 4.5 and the results of Section 3, we immediately have a plethora of conditions equivalent to part (a) of Proposition 4.5.

Proposition 4.6. Let $R=A \oplus B$ as a direct sum of left (right) ideals. If $R$ is adjoint regular, then $A$ and $B$ are adjoint regular rings.

Proof. Let $a \in A$. Then there exist $a_{1} \in A, b_{1} \in B$ such that $a=a \circ\left(a_{1}+b_{1}\right) \circ a=$ $a \circ\left(a_{1}+b_{1}\right)+a-\left[a \circ\left(a_{1}+b_{1}\right)\right] a$. So $a \circ\left(a_{1}+b_{1}\right)=\left[a \circ\left(a_{1}+b_{1}\right)\right] a \in A$. Then $a \circ\left(a_{1}+\right.$ $\left.b_{1}\right)=a+a_{1}+b_{1}-a\left(a_{1}+b_{1}\right)$, or $b_{1}-a b_{1}=a \circ\left(a_{1}+b_{1}\right)-a-a_{1} \in A$. But $b_{1}-a b_{1} \in B$; so $b_{1}-a b_{1}=0$. Then $a=a \circ\left(a_{1}+b_{1}\right) \circ a=\left[a+a\left(a_{1}+b_{1}\right)-a\left(a_{1}+b_{1}\right)\right] \circ a$, or $a=\left(a+a_{1}-a a_{1}\right) \circ a=a \circ a_{1} \circ a$. Proceed similarly for right ideals.

LEMMA 4.7. Let $R$ be adjoint regular.

(a) Either $J(R)=R$ or $R$ contains a nonzero idempotent.

(b) If the module $R$ is indecomposable and $R \neq J(R)$, then $R=e R e \oplus \mathbf{r}(e)$, as a direct sum of right ideals, with eRe a regular ring with identity $e$, and $\mathbf{r}(e)$ is a square zero ideal of $R$. 
(c) If the modules ${ }_{R} R$ and $R_{R}$ are indecomposable, then either $R=J(R)$ or $R$ is a division ring.

Proof. (a) Let $r \in R, r \neq 0$. Then there exists $\bar{r} \in R$ such that $r \circ \bar{r} \circ r=r$, and $r \circ \bar{r}$ and $\bar{r} \circ r$ are nonzero idempotents in $(R, \circ)$. So $r \circ \bar{r}$ and $\bar{r} \circ r$ are also idempotents in $R$. If $R$ has no nonzero idempotent, then $r \circ \bar{r}=\bar{r} \circ r$. So in this case each element of $R$ is quasi-regular, that is, $R=J(R)$.

(b) If $R \neq J(R)$, then there exists a nonzero $r \in R$ such that $e=r \circ \bar{r}$ is a nonzero idempotent in $R$. Then $R=R e \oplus \mathbf{l}(e)$, as a direct sum of left ideals. Since $R e$ and $\mathbf{l}(e)$ are submodules of ${ }_{R} R$, and since $R e \neq 0$, we have $\mathbf{l}(e)=0$, and hence $e$ is a right identity of $R$. So $R=e R \oplus \mathbf{r}(e)$, as a direct sum of right ideals of $R$. However, $R \cdot \mathbf{r}(e)=(R e) \mathbf{r}(e)=0$, so $\mathbf{r}(e)$ is an ideal of $R$ and $\mathbf{r}(e) \subseteq \mathbf{r}(R)$. Since $R$ is adjoint regular, we have that $e R e$ is a regular ring [7, Proposition 2].

(c) Continuing from the proof of (b), since $R_{R}$ is indecomposable and since $e R e \neq 0$, we have that $\mathbf{r}(e)=0$, and hence $e$ is also a left identity. Thus $R=e R e$, a regular ring with identity. Since the ring is indecomposable, either $R=J(R)$ or $R$ is a division ring.

Note that from Lemma 4.7, we have that if $R$ is indecomposable in terms of both left and right ideal decompositions, then either $R$ is a Jacobson radical ring and the regular radical (see [20, Chapter VI]) is zero, or $R$ is equal to its regular radical and the Jacobson radical is zero.

Proposition 4.8. Let $R$ be adjoint regular. If $R$ has a right (left) nonzero semicentral idempotent, then there exist submonoids $A$ and $B$ of $(R, \circ)$ such that

(a) $R=A \circ B$ and $A \cap B=0$;

(b) $R=A \oplus B$ as a direct sum of adjoint regular right (left) ideals of $R$;

(c) $A$ is a regular ring with identity and $B$ is a two-sided ideal of $R$.

Proof. Let $e$ be a nonzero right semicentral idempotent in $R$. Then $R=e R \oplus r(e)$ as a direct sum of right ideals, where $e R=e R e$ is a ring with unity and $r(e)$ is a twosided ideal of $R$. Let $A=e R$ and $B=r(e)$. Observe that $R=A \circ B$ because $A B=0$. By Proposition 4.6, $(A, \circ)$ is regular. Since $A$ is a ring with unity, $A$ is regular. Proceed similarly for $e$ left semicentral.

5. Radicals for adjoint Clifford rings. We show the equivalence of several standard radicals for rings which are adjoint Clifford and obtain a characterization for the Jacobson radical of such rings.

Proposition 5.1. If $R$ is adjoint Clifford, then the Brown-McCoy radical, $\varphi(R)$, and $\mathscr{f}(R)$ are equal.

Proof. For purposes of contradiction suppose that there is an adjoint Clifford ring $R$ with $\mathscr{g}(R) \neq \mathscr{G}(R)$. Then $R / \mathscr{f}(R)$ is also adjoint Clifford and $\mathscr{f}(R / \mathscr{f}(R))=0$. So, without loss of generality, take $\mathscr{g}(R)=0$. Since $R$ is adjoint Clifford it is a subdirect product of division rings by [13, Proposition 3.7]. Because $\mathscr{G}(R) \neq 0$, at least one of these homomorphic image division rings must have nonzero Brown-McCoy radical, a contradiction. 
EXAMPLE 5.2. Proposition 5.1 cannot be extended to all adjoint regular rings, even if the ring is also adjoint simple, as the following example illustrates. Let $V$ be a vector space over a field $F$ with $\operatorname{dim}_{F} V=\aleph_{\omega}$, and let $\omega$ be the first infinite limit ordinal. Let $R=\left\{\phi \in \operatorname{End}_{F} V \mid \operatorname{rank} \phi<\aleph_{\omega}\right\}$. It is well known that $R$ is a regular ring with no maximal ideal. So $J(R)=0$ and $G(R)=R$. It is also known that $(R, \circ)$ is simple, [4, Example 3B].

In the next proposition, when $R$ does not have unity we use the unity element in the Dorroh extension for convenience of expression.

Proposition 5.3. If $R$ is adjoint Clifford, then $\mathscr{g}(R)=\cap_{e \in E}(1-e) R$.

Proof. Let $R=e R \oplus(1-e) R$ as ideals. Then $e R$ is adjoint Clifford because the map $\phi: R \rightarrow e R$ is a ring homomorphism and so $\phi:(R, \circ) \rightarrow(e R, \circ)$ is a semigroup homomorphism. Therefore $\mathscr{g}(R) \subseteq(1-e) R$. Since $e$ is arbitrary, we have that $\mathscr{g}(R) \subseteq$ $\cap_{e \in E}(1-e) R$.

Conversely, let $I=\cap_{e \in E}(1-e) R$. Then $I$ is an ideal, hence adjoint Clifford. But $I$ contains no nonzero idempotent. Therefore $(I, \circ)$ is a group, so $I \subseteq \mathscr{g}(R)$.

\section{REFERENCES}

[1] A. A. Albert, Structure of Algebras, American Mathematical Society Colloquium Publications, vol. 24, American Mathematical Society, New York, 1939.

[2] G. F. Birkenmeier, Idempotents and completely semiprime ideals, Comm. Algebra 11 (1983), no. 6, 567-580.

[3] W. E. Clark, Generalized radical rings, Canad. J. Math. 20 (1968), 88-94.

[4] W. E. Clark and J. Lewin, On minimal ideals in the circle composition semigroup of a ring, Publ. Math. Debrecen 14 (1967), 99-104.

[5] A. H. Clifford and G. B. Preston, The Algebraic Theory of Semigroups. Vol. I, Mathematical Surveys, no. 7, American Mathematical Society, Rhode Island, 1961.

[6] M. P. Drazin, Rings with central idempotent or nilpotent elements, Proc. Edinburgh Math. Soc. (2) 9 (1958), 157-165.

[7] X. K. Du, The rings with regular adjoint semigroups, Northeast. Math. J. 4 (1988), no. 4, 463-468.

[8] _ The structure of generalized radical rings, Northeast. Math. J. 4 (1988), no. 1, 101-114.

[9]__ The adjoint semigroup of a ring, preprint, 2001.

[10] L. Fuchs and I. Halperin, On the imbedding of a regular ring in a regular ring with identity, Fund. Math. 54 (1964), 285-290.

[11] K. R. Goodearl, Von Neumann Regular Rings, Monographs and Studies in Mathematics, vol. 4, Pitman, Massachusetts, 1979.

[12] T. E. Hall, On regular semigroups, J. Algebra 24 (1973), 1-24.

[13] H. E. Heatherly and R. P. Tucci, Adjoint Clifford rings, to appear in Acta Math. Hungar.

[14] _ The circle semigroup of a ring, Acta Math. Hungar. 90 (2001), 231-242.

[15] N. Jacobson, Structure of Rings, American Mathematical Society Colloquium Publications, vol. 37, American Mathematical Society, Rhode Island, 1968.

[16] L. Kovács, A note on regular rings, Publ. Math. Debrecen 4 (1956), 465-468.

[17] J. O. Loyola, e-free objects in e-varieties of inverse rings, Semigroup Forum 54 (1997), no. 3, 375-380.

[18] J. Luh, A note on strongly regular rings, Proc. Japan Acad. 40 (1964), 74-75.

[19] M. Petrich and N. R. Reilly, Completely Regular Semigroups, Canadian Mathematical Society Series of Monographs and Advanced Texts, vol. 23, John Wiley \& Sons, New York, 1999. 
[20] F. A. Szász, Radicals of Rings, John Wiley \& Sons, Chichester, 1981.

[21] P. G. Trotter, Congruence extensions in regular semigroups, J. Algebra 137 (1991), no. 1, 166-179.

[22] J. Zeleznikov, Orthodox rings and semigroups, J. Austral. Math. Soc. Ser. A 30 (1980), $50-54$.

HeNRy E. HEATHerly: Department of MATHEMATics, University of LOUisiana at LAFAYETTE, LAFAYETTE, LA 70504-1010, USA

Ralph P. Tucci: Department of Mathematics and Computer Science, loyola UNIVERSITY, NEW ORLEANS, LA 70118, USA

E-mail address: tucci@1oyno.edu 


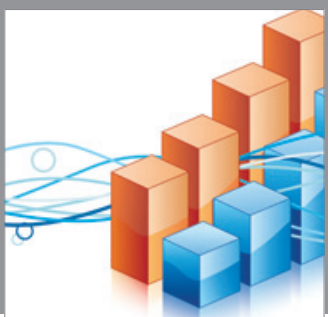

Advances in

Operations Research

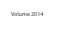

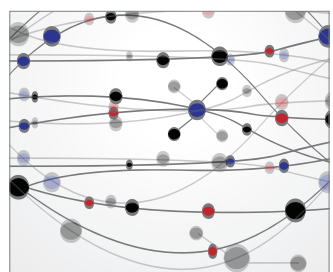

\section{The Scientific} World Journal
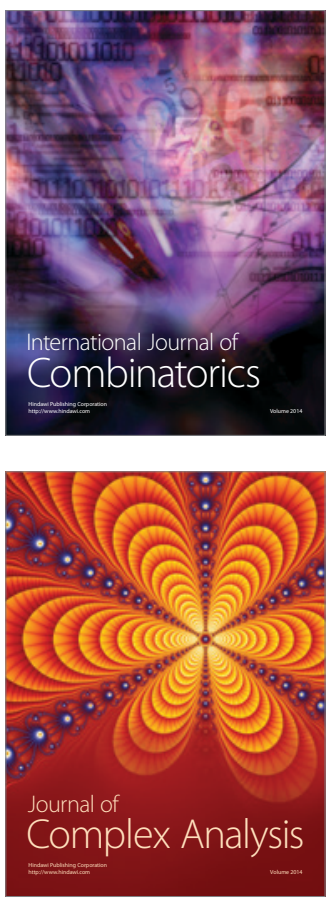

International Journal of

Mathematics and

Mathematical

Sciences
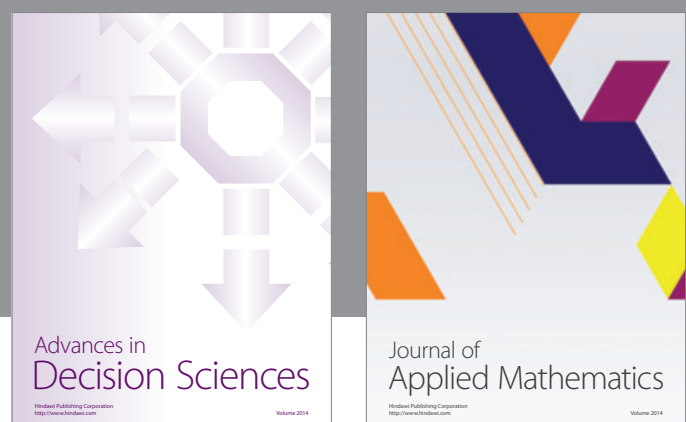

Journal of

Applied Mathematics
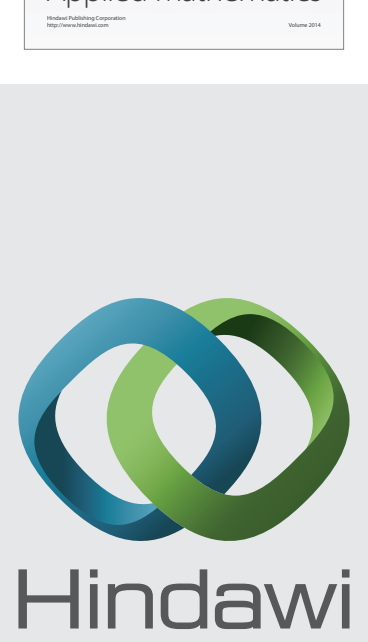

Submit your manuscripts at http://www.hindawi.com
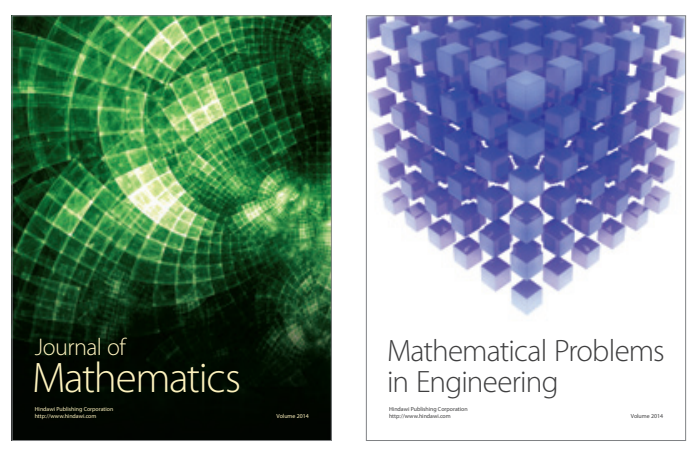

Mathematical Problems in Engineering
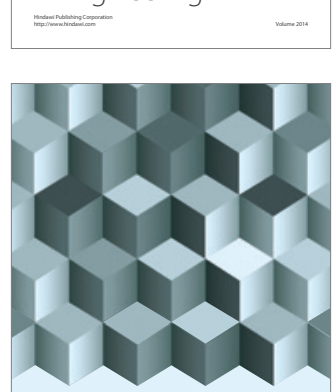

Journal of

Function Spaces
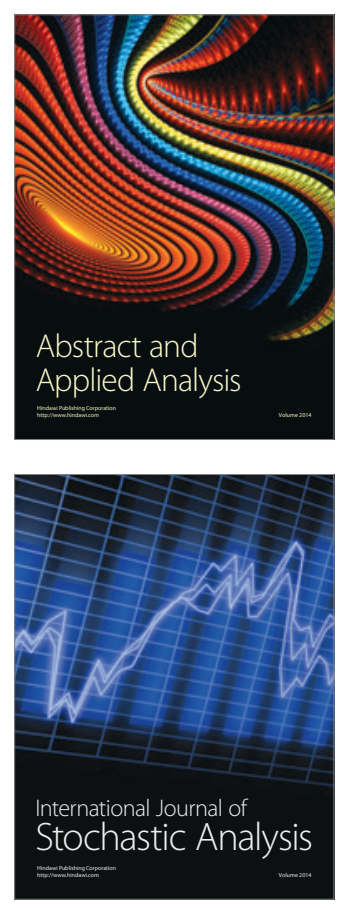

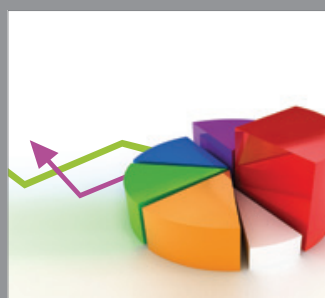

ournal of

Probability and Statistics

Promensencen
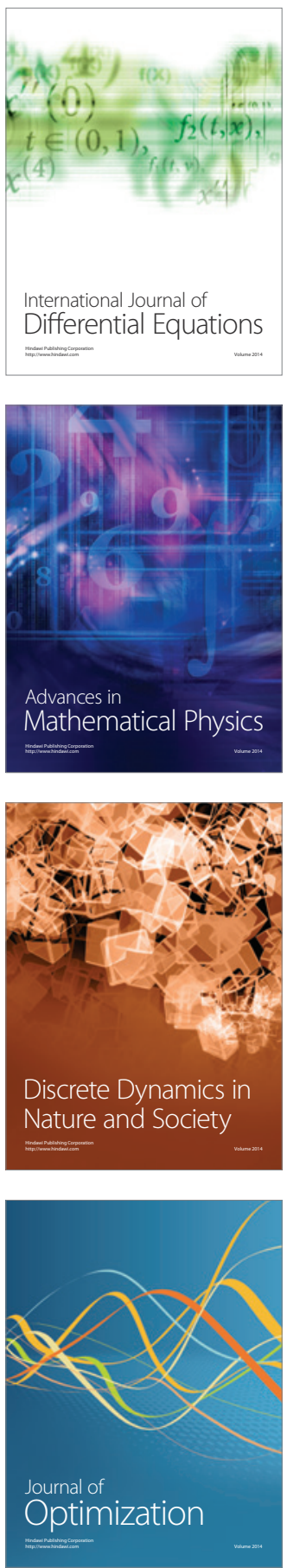